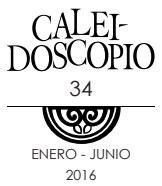

\title{
Instituciones en adicciones "la organización y la transferencia de tecnologías en psicología"
}

ALEJANDRA OREJEL BERMÚDEZ'

CECILIA MÉNDEZ SÁNCHEZ²

\section{RESUMEN}

El estudio de las organizaciones y su análisis es de gran utilidad para diferentes fines, entre éstos favorecer o lograr la transferencia de tecnologías. En la actualidad, en el ámbito de la salud, específicamente de las adicciones, se busca la transferencia de tecnologías en psicología, que son programas de intervención para la atención y prevención de adicciones en las 310 Unidades Médicas de Especialidad-Centros de Atención de Prevención de Adicciones de México. Ante esta situación, se buscó hacer las primeras aproximaciones al análisis de las Unidades, a través del conocimiento del clima organizacional por medio del Cuestionario de clima laboral (Corral y Pereña, 2003) de dos Unidades, encontrando que a pesar de que siguen un mismo sistema, tienen diferente clima organizacional según los resultados de la aplicación, los cuales se podrían explicar por algunas variables observadas en cada unidad de trabajo.

Palabras clave: análisis organizacional, clima organizacional, transferencia de tecnología, instituciones de adicciones, condiciones laborales.

1 Licenciada en psicología por la Universidad Autónoma de Aguascalientes. Colaboradora de investigación en el Departamento de Psicología, Universidad Autónoma de Aguascalientes. psico.aleorejel@hotmail.com.

2 Doctora en cognición y trastornos por la Universidad Autónoma de Madrid. Profesora e investigadora de dedicación parcial 40 hrs., titular C. Universidad Autónoma de Aguascalientes.cmendez@correo.uaa.mx. 


\section{ABSTRACT}

The study of organizations and their analysis is useful for different purposes, between those in favor or achieve technology transfer. Currently in the field of health addictions, specifically seeks the transfer of technologies that are psychological intervention programs for the treatment and prevention of addiction in the medical units of 310 Specialty Care Centers for Addiction Prevention in Mexico, in this situation we sought to make the first approaches to the analysis of the Centers through knowing the organizational climate through the Environmental Working Questionnaire (Corral and Pereña, 2002) of two Centers, finding that although they follow the same system, they have different organizational climate according to the results of the application, which could explain some variables observed in each unit.

Key words: organizational analysis, organizational climate, technology transfer, institutions of addiction, working conditions.

\section{LAS ADICCIONES Y EL USO DE TECNOLOGÍAS EN PSICOLOGÍA PARA SU PREVENCIÓN EN INSTITUCIONES DE MÉXICO}

La temática del abuso de consumo y adicción a las drogas de tipo legal como el alcohol, el tabaco y las drogas ilegales en su gran diversidad de presentaciones se ha vuelto un tema de interés en nuestro país, debido a que, al igual que otros países, se ha encontrado un aumento de incidencia de consumo en edades más tempranas y drogas más dañinas (ENA, 2011), además de que el abuso o dependencia a estas sustancias conllevan consecuencias que generan problemas sociales, sanitarios y en quien las consume.

Por tanto, el país se enfrenta a una problemática real, que necesita de una o varias alternativas efectivas para favorecer la solución. Ante esto, ¿qué solución tiene la psicología? Una de ellas son las tecnologías de tipo preventivas, entendiendo como tecnologías no a los artefactos, como computadoras, maquinaria, etc., que solemos evocar cuando escuchamos la palabra "tecnología", sino más bien refiriéndonos a los procesos o formas de hacer cierta tarea, orientados a lograr la solución de problemas producto de las ciencias (Vercelli, 2009).

En este caso, las tecnologías preventivas de la psicología son tratamientos protocolizados desde el modelo cognitivo-conductual, para 
fomentar el cambio en el patrón de consumo de diversas drogas en usuarios no dependientes, pero que reportan problemas a causa de su forma de consumo. Estos programas lograron asegurar su efectividad a partir de la investigación científica, cuya finalidad es ofrecer una práctica clínica de mayor calidad, eficacia y con el menor costo posible (Gutiérrez y González, 2010).

La existencia en sí misma de estos programas no cambia la realidad del problema de las adicciones, pero sí su utilización de manera apropiada a la mayor cantidad de personas que los requieren; para esto, la Comisión Nacional Contra las Adicciones (CONADIC) busca que se utilicen en las 310 Unidades Médicas de Especialidad-Centros de Atención de Prevención de Adicciones (UNEME-CAPA) ubicados en todo el país, para que logren cumplir, de acuerdo a las mejoras alternativas existentes, su objetivo de " [... ] la prevención, tratamiento y formación de personal para el control de adicciones, con el propósito de mejorar la calidad de vida individual, familiar y social de la población" (CONADIC, 2010).

A pesar de que estos programas son accesibles a través de manuales en los UNEME-CAPA, investigaciones actuales como la de Reyes (2012) y la de Vargas, Martínez, Lira y Oropeza (2014), han encontrado que en ocasiones no se aplican íntegramente o no se utilizan, lo que pone en riesgo la efectividad tanto de las tecnologías como del logro del objetivo de la institución con la calidad esperada. Ante esta situación, encontramos que el conflicto de la aún no adopción de los programas en los UNEME-CAPA, nos refleja una dificultad para conseguir la transferencia de las tecnologías de manera eficiente.

\section{¿QuÉ SE CONOCE DE LA ORGANIZACIÓN DE LAS UNIDADES MÉDICAS de Especialidad-CENTROS de Atención de PREVEnCIÓN DE ADICCIONES (UNEME-CAPA) Y LA RELEVANCIA DE SU ESTUDIO?}

Las UNEME-CAPA son producto de un proyecto que inicia en el año 2007, sustentado económicamente por el decomiso del narcotráfico y regulado por la NOM-028-SSA2-2009. Estas unidades y centros cuentan con una infraestructura con sala de espera, áreas de trabajo, salón de usos múltiples y con personal conformado por directivos, psicólogos, médicos, trabajadores sociales, recepcionistas, personal administrativo y de mantenimiento (CONADIC, 2010). 
Los servicios se brindan de manera gratuita, y son: detección temprana a jóvenes con vulnerabilidad a dependencia en escuelas; intervención breve para disminuir la prevalencia de abuso a sustancias en adolescentes y adultos; diagnóstico bio-psico-social a quienes padecen una adicción; apoyo a las familias de experimentadores, abusadores o dependientes; canalización oportuna; prevención con infantes; prevención de recaídas; seguimiento de casos; talleres de sensibilización y temáticas que ayuden afrontar las situaciones de consumo; talleres de crianza positiva; orientación preventiva; favorecer investigaciones como apoyo a un sistema de vigilancia epidemiológica; y apoyo y conformación de grupos de ayuda mutua (CONADIC, 2010).

Por otro lado, también conocemos datos de la organización obtenidos a través del estudio de Martínez, Pacheco, Echeverría y Medina-Mora (en prensa), cuyo fin es identificar las barreras de la adopción de los programas a partir de entrevistas a los actores que están implicados en la misma (investigadores, terapeutas y directivos del UNEME-CAPA), donde se encontró que existe una falta de recursos, diversidad en la formación de los terapeutas, la cual en ocasiones no compagina con el modelo de los programas, altas demandas de la institución hacia los terapeutas y trámites burocráticos que obstaculizan las actividades de los mismos. También reportan que dentro de las opiniones del personal de los UNEME-CAPA acerca de las tecnologías, se encuentran dos tipos: las opiniones positivas, que las consideran herramientas útiles; y las negativas, que las consideran una limitación al juicio y autonomía profesional que se transmite a través de la imposición (Martínez y Medina-Mora, 2013).

Estos datos aún no son suficientes para hacer un análisis de la situación social de los UNEME-CAPA; para una exploración más profunda es necesario analizar las interacciones de los elementos que componen la institución donde se entremezclarán los factores objetivos y los factores subjetivos de los actores de la institución. Ante los cambios sociales y laborales, ante la posibilidad de que el personal que está inmerso en los escenarios de salud y sus condiciones laborales dificulten el logro de sus objetivos, pues su trabajo está promoviendo que en muchos casos no se encuentren en la mejor disposición para realizar sus actividades, que genere repercusión en su servicio y atención a los usuarios, además de que ésta pueda generar barreras o poca disposición del personal para la aceptación de tecnologías. Significaría enfrentarnos a una situación necesaria de atender, ya que 
comprender más este aspecto nos permitiría proponer otras soluciones para el logro de la transferencia, favorecer la higiene social de esas organizaciones y, de manera indirecta, favorecer a la mejora de la calidad de servicio para los beneficiarios de esta institución.

Por todo lo anterior, el objetivo del presente trabajo fue realizar las primeras aproximaciones al análisis de los UNEME-CAPA a través del conocimiento de su clima organizacional, a fin de encontrar información de la percepción que tienen del mismo el personal, así como encontrar datos que puedan ser relevantes para encontrar y/o entender situaciones que ocasionan obstáculos para la transferencia de los programas de intervención psicológicos.

MÉTODO

\section{Participantes}

El estudio fue realizado con el total del personal que conforman dos UNEME-CAPA ( 21 empleados). El UNEME-CAPA 1 está situado en una zona rural y el UNEME-CAPA 2 en una zona urbana. Se desglosan las características de cada una de las personas del estudio en la Tabla 1. 


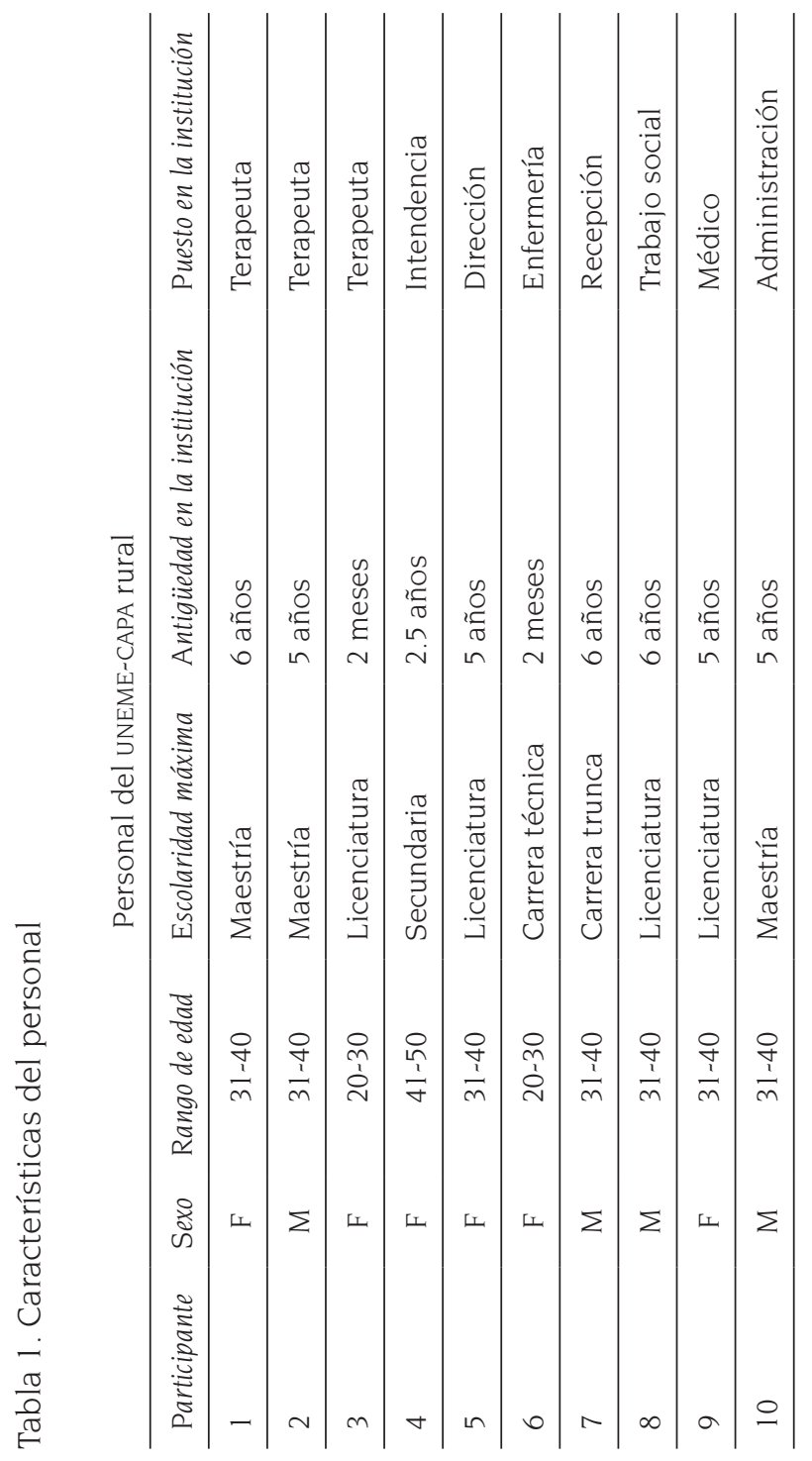




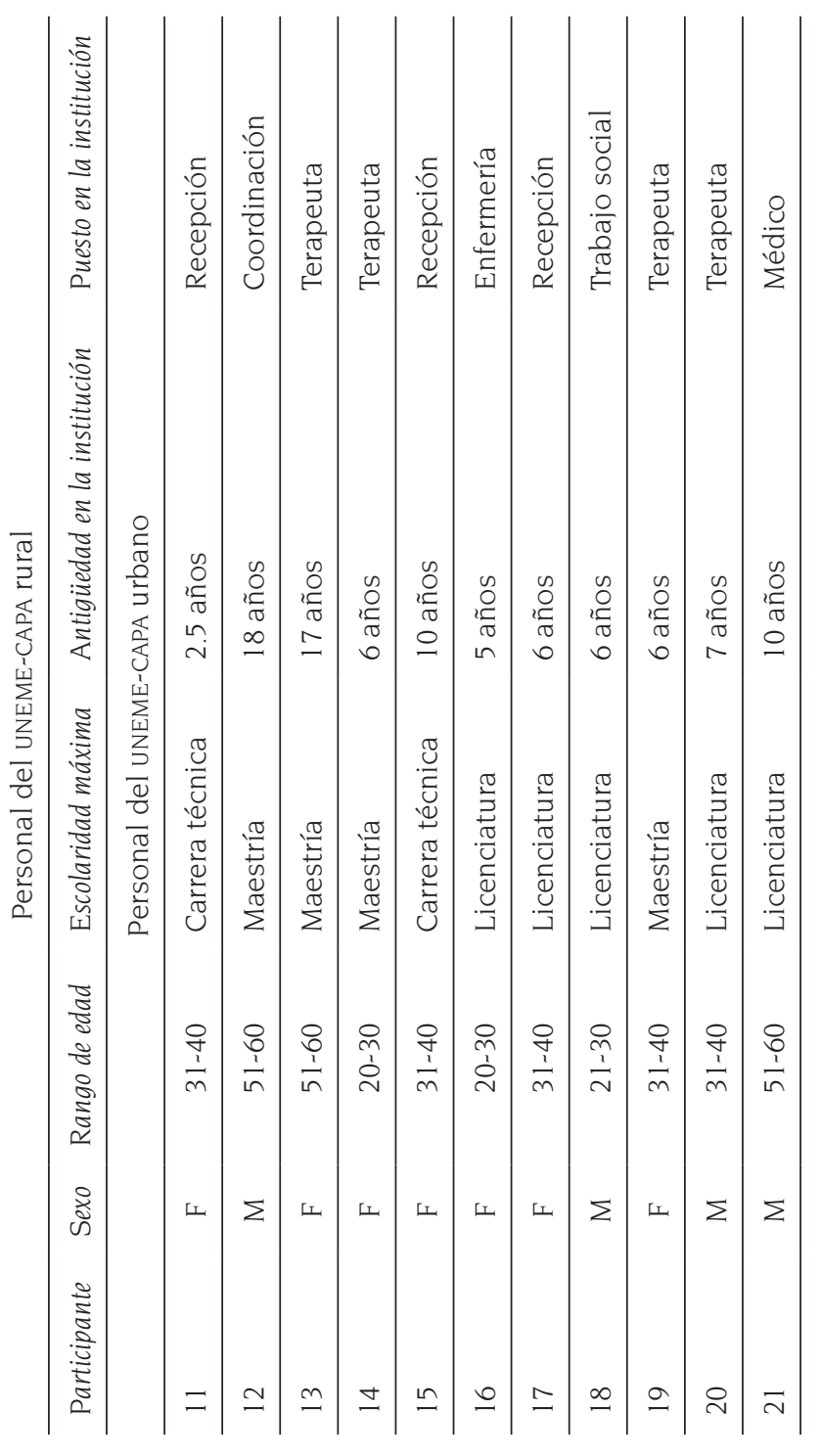

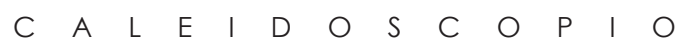




\section{INSTRUMENTOS}

\section{Cuestionario de Clima Laboral (CLA) de Corral y Pereña (2003)}

El Cla es un cuestionario que tiene el objetivo de evaluar el clima organizacional. Consta de 93 ítems de frases cortas que reflejan comportamientos de los trabajadores que pueden afectar a la percepción y valoración de la organización en general; considera tres opciones de respuesta: sí, incógnita y no. Estos ítems son indicadores de 8 dimensiones de dos ejes, el primer eje es empresa u organización que refleja lo dependiente al funcionamiento y organización de la institución, y sus dimensiones son: Innovación, Información, Condiciones y Organización; y el segundo eje corresponde a las vivencias y actitudes del trabajador en la organización con las dimensiones: Implicación, Relaciones, Autorrealización y Dirección.

\section{PROCEDIMIENTO}

El estudio se diseñó usando una metodología de tipo cuantitativa a través de la aplicación del cuestionario CLA y su análisis, diferenciado por institución y uno comparativo. A continuación se describe el procedimiento empleado:

1. Se realizó el estudio en dos UNEME-CAPA, los cuales se seleccionaron de manera intencionada por cuestiones sociodemográficas.

2. Se decidió valorar el clima organizacional a partir de la implementación de un instrumento, resultando seleccionado el Cuestionario de Clima Laboral.

3. Posteriormente, se contactó a las instituciones a través del directivo y se les solicitó el permiso de la aplicación.

4. Se aplicó el cuestionario CLA a todo el personal de ambas instituciones, la resolución del cuestionario era de manera anónima y durante la aplicación se aseguró que quedara claro el objetivo del instrumento, y se promovió la honestidad en su resolución.

5. Para el análisis de este cuestionario se utilizó un programa computacional. Para esta aplicación, en un primer momento, se analizó la dirección del reactivo, es decir, si éste era positivo o negativo para otorgarle un valor a cada respuesta, resultando tener un valor de dos puntos la respuesta que indicaba una cua- 
lidad positiva del clima organizacional, un punto la respuesta que no aseguraba algo negativo ni positivo del clima y 0 puntos si la respuesta reflejaba una cualidad negativa de la institución.

6. También los ítems del instrumento se clasificaron en las 8 diferentes dimensiones a través de un análisis interjueces, siendo los jueces 3 profesionales en psicología que utilizaron los conceptos otorgados por el instrumento para la clasificación.

7. Los resultados de los cuestionarios se vaciaron y se realizó un análisis de tipo descriptivo por cada institución, así como una comparación de ambos grupos por medio de ANOvA, de un factor donde se consideró una diferencia significativa, cuando $p$ era igual o menor a 0.05 a través del programa SPSS.

\section{RESULTADOS}

A manera general, se encontró que el clima organizacional es más adecuado en el UNEME-CAPA rural que en el urbano, esto en relación con todas las dimensiones, como se puede apreciar en la Figura 2.

Figura 2. Resultados generales por dimensión del clima organizacional.

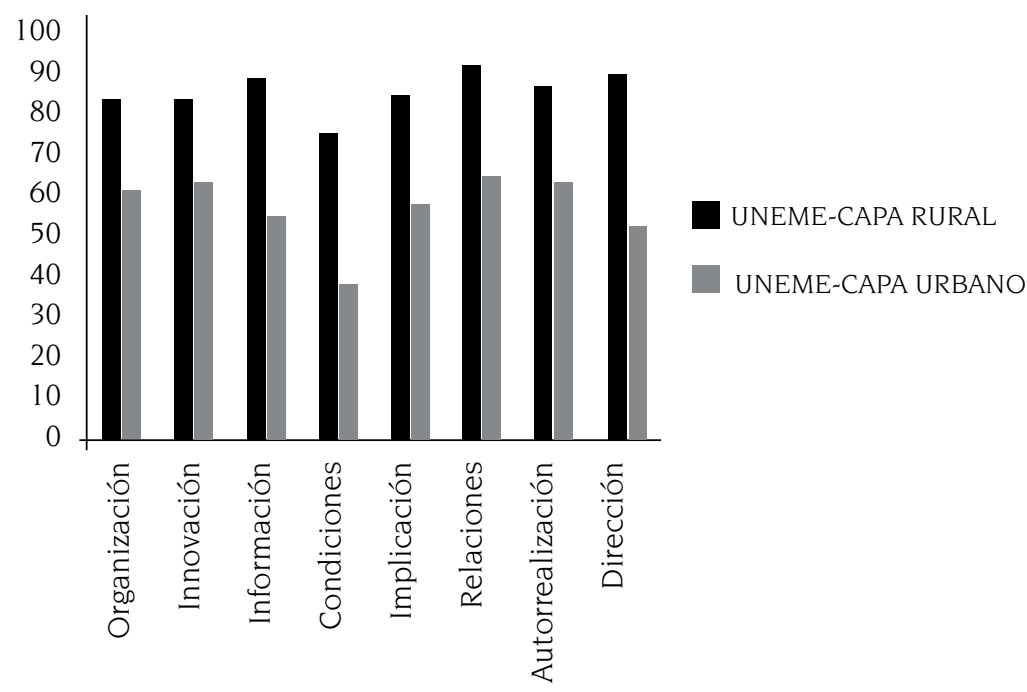

* De manera más específica, de las 8 dimensiones del clima organizacional, se reportó, respecto a la dimensión de organización, que ambas reflejan una alta claridad en 
cuanto a los roles y distribución de tareas en la institución, además de que existe una planificación de las actividades, pero también ambas instituciones consideran que la organización no reconoce apropiadamente los esfuerzos del personal; a su vez, concuerdan que los procesos burocráticos afectan negativamente el desarrollo de su trabajo, pero esta percepción es más grave en el urbano $(\mathrm{p}<0.05)$. Además, se encuentra una diferencia estadística significativa en relación a la comprensión y claridad que se tiene de los privilegios que gozan los superiores, ya que el rural se reporta más conforme al respecto.

Acerca de la dimensión de innovación, ambas instituciones consideran que en el ámbito laboral haber trabajado en la institución es una buena referencia, pero que su trabajo no les otorga ventajas sociales satisfactorias. Sin embargo, ambas instituciones tienen diferencias significativas respecto a la visión de rigidez y tradicionalismo, ya que para el urbano esto es una característica de la organización que para el rural no lo es, además, es estadísticamente significativo que el urbano reporta constantes problemas en la institución por falta de calidad, los cuales no reporta el rural. Ambas coinciden en que sus métodos de trabajo y actividades están medianamente actualizados y son diversos, pero difieren acerca de la percepción del dinamismo en sus instituciones y la utilidad de todas las tareas desempeñadas, siendo una visión más positiva en el rural.

En la dimensión de información, ambas instituciones reflejan conocimiento de qué se hace en otros departamentos; sin embargo, se reportaron varias diferencias estadísticamente significativas en esta área, como fue que para el urbano la información no circula con fluidez, que coincide con la percepción de que no es de dominio popular, por lo que les es difícil obtener información fiable, aunado a que no perciben esfuerzos o intento de mejorar los canales de comunicación.

Respecto a la dimensión de condiciones, en ambas instituciones fue la que más bajos resultados obtuvo, pero los del urbano reportan una situación más alarmante en esta área. Ambas consideran que sus remuneraciones no son satisfactorias; respecto a las condiciones materiales, ambas reflejan la necesidad de mejorar sistemas informáticos, pero difieren en los otros aspectos, ya que para el rural éstos gozan de instalaciones buenas y en general de materiales que permiten el desarrollo que de manera estadísticamente significativa no perciben o gozan los del urbano.

En implicación se encontró que ambas instituciones difieren significativamente, ya que para el urbano el trabajo se practica individualmente, 
además de que no suelen ser implicados en las decisiones que se toman y no se favorece la cooperación para que se den los cambios, a diferencia de rural, aunque tanto rural como urbano consideran que la gente espera salir con ansias del trabajo, que existe iniciativa y que el éxito y eficacia está directamente influenciado con su labor, aunque por otro lado, el urbano reporta que el trabajo se realiza frecuentemente con desgano, sin embargo, ambos concuerdan en que no se tiene gran control acerca del ritmo y los objetivos de trabajo.

De acuerdo con los resultados obtenidos, las relaciones sociales, en el rural son su área de mayor fortaleza, iniciando porque percibe un ambiente social más cálido que el urbano, aunque también ambas advierten que son accesibles para apoyar al personal nuevo que ingresa, pero se encontraron diferencias significativas en cuanto a que el rural considera que se da la expresión libre de ideas, de manera cotidiana las conversaciones personales, que no existe la necesidad de sabotear y que es fácil obtener el apoyo de los superiores, lo anterior se presenta de manera contraria en el urbano.

En relación con la dimensión de autorrealización, el personal de ambas instituciones refiere que ha tenido nuevos aprendizajes gracias a su trabajo dentro de las mismas y que perciben cierta libertad para hacer sus tareas, además de que les interesa su trabajo; por lo contrario, difieren significativamente en la percepción de oportunidades de formación y la posibilidad de progresar dentro de la misma institución.

Por último, respecto a la percepción en la dimensión de dirección, se encontró que en esta área la mayoría de los aspectos de la misma reflejan diferencias estadísticamente significativas, siendo un panorama más positivo en el rural; entre las diferencias está que el urbano percibe que las decisiones se toman sin tomar en cuenta los intereses de otros, además de que no existe una claridad de las razones de las mismas, por lo que también se percibe que éstas no son las más eficaces, además de que la aportación de ideas no se estimula o se aprecia, por lo que encuentran poca accesibilidad a realizar cambios aunque sean necesarios, ambos coinciden en que los ascensos se presentan por otra situación ajena al servicio y antigüedad, y que las decisiones tomadas en ocasiones no consideran el trabajo previo.

Otro aspecto importante fue lo observado durante la recopilación de datos; en el UNEME-CAPA urbano gran parte del personal realiza Comentarios, cuestiona el motivo del cuestionario y si los resultados podrían favorecer cambios, ya que observaban que eran necesarios, 
manifestaban un momento para expresar su inconformidad realizando comentarios de su situación en la organización y de una situación general. En el rural, se observó un inconveniente para iniciar la aplicación por dificultad de encontrar al directivo, además éste era muy positivo al acceder a las peticiones, pero posteriormente prohibía que se realizaran ciertos procedimientos que incluyeran el trabajo que se realizaba, durante las visitas se observa que el personal suele relacionarse de manera fluida y con comadrería, a excepción del médico y administración que permanecen aislados con actitud seria, la sala de espera permanece vacía o con una sola persona y el personal goza de tiempo sin actividad.

\section{DISCUSIÓN}

Como se observa en los resultados de este estudio, a pesar de que ambas unidades tienen el mismo sistema organizacional o deberían tenerlo, hay diferencias en el clima organizacional, aunque también es posible encontrar bondades y aspectos a mejorar compartidos, además de que el conocimiento de esos puntos compartidos permitirán que se planteen estrategias más apropiadas para el logro de la transferencia de los programas de intervención; sin embargo, por el momento este estudio es una de las primeras aproximaciones y esfuerzos para realizar un análisis de estas instituciones.

En este caso, la diferencia en el clima en ambas unidades podría tener relación con distintos aspectos como el diferente grado de demanda social que se tiene, ya que la presión de atención en el urbano es considerablemente más grande, pues en la otra unidad la menor demanda favorece que se tengan tiempos de descanso donde pueden aprovechar para establecer relaciones sociales, o realizar otras tareas. La fortaleza que tiene en cuanto a relaciones sociales el UNEME-CAPA rural, las condiciones físicas y materiales más favorables, además de que el personal goza de menor cantidad de años de trabajo, hacen que sean menos proclives a padecer síndrome de burnout, a diferencia del urbano.

Otro aspecto a considerar es la forma de relacionarse con la institución de salud mental a nivel espacial y direccional, que hace que la interacción sea menos próxima y específica, por lo que esto puede afectar los canales de comunicación y la inconformidad que se tiene con la forma de dirección. 
Estos primeros resultados nos indican que las condiciones laborales de la institución, aunque no son altamente riesgosas, tienen aspectos que pueden mejorarse en beneficio del personal, pero también es importante señalar que para futuros estudios es esencial agregar como estrategia la observación directa para recabar datos objetivos de la situación, también sería importante realizar un análisis por puesto y ampliar la muestra de centros, para así poder lograr realizar un diagnóstico de los UNEME-CAPA.

Además, al igual que Brown (2000), se considera que el análisis institucional es una tarea fundamental para poder entender los obstáculos actuales que se han tenido en la transferencia, y también es importante que se hagan en la medida de lo posible modificaciones a nivel organizacional que incidan positivamente en el personal y su calidad de vida, además de que favorezcan el logro exitoso de la transferencia.

\section{REFERENCIAS}

Brown, B.S. (2000). From research to practice - The bridge is out and the water's rising. En J. A. Levy, R. C.Stephens, y D.C. McBride (Eds.). Emergent issues in the field of drug abuse. Advances in medical organizations and systems (pp. 345-365). Standford: JAI Press.

CONADIC. (2010). Recuperado de: http://www.conadic.salud.gob.mx/ nueva_vida/pres_nuevavida.html.

Corral, S. y Pereña, J. (2003). CLA. Cuestionario de clima laboral. Manual del Work Climate Questionnaire. Madrid: Ediciones TEA.

ENA. (2011). Recuperado de: http://www.cenadic.salud.gob.mx/PDFS/ investigacion/ ENA2011_alcohol.pdf.

Gutiérrez, I. y González, C. (2010). ¿Cómo localizar GPC? Recuperado el 2 de marzo del 2014 de: http://www.fisterra.com/guias2/fmc/localizar. asp.

Martínez Martínez, K. I., y Medina-Mora Icaza, M. E. (2013). Transferencia tecnológica en el área de adicciones: el programa de intervención breve para adolescentes, retos y perspectivas. Salud mental, 36(6), 505-512. Recuperado el 27 de enero de 2016 de: $\quad$ http://www.scielo.org.mx/scielo.php?script=sci_arttextEpi$\mathrm{d}=$ S018533252013000600008\&lng=es\&tlng=es.

Martínez, M. K. I., Pacheco, T. Y. A., Echeverría, L. y Medina-Mora, M. E. (en prensa). Revista de Salud Mental. 
Reyes, M. (2012). Integridad de tratamiento de un programa validado empíricamente en terapeutas que laboran en escenarios clínicos. Tesis de maestría. Universidad Autónoma de Aguascalientes.

Vargas, E., Martínez, K., Lira, J. y Oropeza, R. (2014). Factores que determinan la adopción de la práctica con base en la evidencia en terapeutas especialistas en adicciones en México. $2^{\circ}$ Coloquio Internacional de Analistas Conductuales Aplicados. Autocontrol y Adicciones. México, D.F.

Vercelli, A. (2009). Reconsiderando las tecnologías sociales como bienes comunes. Íconos Revista de Ciencias Sociales de FLACSO Ecuador.

(3) 